\title{
Uso Ético del Poder Gerencial: Propuesta de un Programa para la Formación de Estudiantes de Ingeniería
}

\author{
María del P. Rodríguez ${ }^{(1)}$, Martín A. Pantoja ${ }^{(1)}$ y Fernando Cantor ${ }^{(2)}$ \\ (1) Facultad de Ingeniería y Arquitectura, Dpto. de Ingeniería Industrial, Universidad Nacional de \\ Colombia - Sede Manizales, Campus La Nubia, Manizales-Colombia \\ (e-mail: mdrodriguezco@unal.edu.co; mapantojao@unal.edu.co) \\ (2) Facultad de Ciencias Jurídicas y Sociales, Dpto. de Antropología y Sociología, Universidad de Caldas, \\ Campus Palogrande, Manizales - Colombia (e-mail: fernando.cantor@ucaldas.edu.co)
}

Recibido Sep. 22, 2015; Aceptado Nov. 11, 2015; Versión final Feb. 1, 2016, Publicado Jun. 2016

\begin{abstract}
Resumen
El artículo propone un programa de formación en uso ético del poder gerencial para estudiantes de ingeniería. La metodología utilizada es cualitativa y siguió cinco etapas: conceptual, relacional, analítica, creativa y empírica. En las dos primeras etapas se construyó el marco teórico, en la tercera se seleccionó el enfoque pedagógico, en la cuarta se diseñó el programa y en la quinta se evaluó su implementación. La propuesta se enmarca en la pedagogía constructivista social, y el programa combina sesiones teóricas y lecturas con actividades experienciales y lúdicas. Se concluye que la pedagogía escogida es la más adecuada para las necesidades de formación, ya que permite la introyección de la fundamentación teórica a través de actividades relacionadas con casos de estudio, asuntos trágicos, análisis de la literatura y vivencias personales. Asimismo, el programa contribuye a desarrollar competencias para el uso ético del poder asociado a la práctica ingenieril, y puede ser transferido a otras facultades de ingeniería.
\end{abstract}

Palabras clave: ética; formación de estudiantes de ingeniería; gerencia; poder; uso ético del poder

\section{Ethical use of Managerial Power: A Proposal of an Academic Program for the Training of Engineering Students}

\begin{abstract}
This paper proposes a training program for the ethical use of managerial power for engineering students. The methodology used is of qualitative-type and included five stages: conceptual, relational, analytical, creative and empirical. In the two first stages the theoretical framework was build, in the third the pedagogical approach was selected, in the fourth the program was designed and in the fifth the program was tested. The program follows a social constructivist pedagogy approach and mixes theoretical sessions and readings with experiential and ludic activities. It is concluded that the proposed pedagogical approach is the most suitable for the training needs, because it allows the introjection of theoretical foundations through activities related with case studies, tragic issues, literature analysis, and personal experiences. Furthermore, the program contributes to develop competencies for the ethical use of power associated to engineering practice, and can be transferred to other engineering schools.
\end{abstract}

Keywords: ethics; engineering students training; management; power; ethical use of power 
Uso Ético del Poder Gerencial: Propuesta de un Programa para la Formación de Estudiantes Rodríguez

\section{INTRODUCCIÓN}

Entre las personas decisivas para el buen desempeño económico, social y ambiental de las organizaciones están los gerentes. Son ellos los encargados de realizar tareas como modelar el ambiente de trabajo, formular una visión estratégica, asignar recursos, formar gerentes de primera, fortalecer la organización y realizar la administración cotidiana (Pearson, 1995). Aunque para algunos autores como Kemp (2013) dichas tareas están contenidas en las planteadas por Fayol (1949) en su Teoría Clásica de la Administración (planear, organizar, dirigir y controlar), aún persiste el debate en los estudios gerenciales sobre lo que hacen los gerentes (Mintzberg, 2004; Oswick et al., 2011). En lo que si hay consenso es que uno de los requisitos para el éxito del trabajo de los gerentes es que estos hagan un uso adecuado del poder conferido por las organizaciones, lo que no es extraño si se comparte con Weber (1977) que las organizaciones son estructuras en las cuales se expresa el poder, entendido como "la probabilidad de imponer la propia voluntad, dentro de una relación social, aun contra toda resistencia y cualquiera que sea el fundamento de esa probabilidad" (Weber, 1977, p. 43). En otras palabras, el poder es aquella capacidad para hacer que alguien haga algo que de otra forma no estaría dispuesto a hacer.

Según Galbraith (1985) el poder proviene de tres fuentes: la personalidad, la propiedad y la organización, las cuales, respectivamente, se encuentran asociadas a un instrumento: poder condigno, poder compensatorio y poder condicionado. La personalidad es la fuente más primitiva a la que se ha asociado el origen del poder. En la Antigüedad, las personalidades de los grandes dirigentes eran tomadas como ejemplos para ilustrar no solo el origen, sino también las formas en las que se ejercía el poder. Estas personalidades cargadas de rasgos distintivos impactaban por su carisma, aquella cualidad especial que otorga a una persona la capacidad para atraer y fascinar con su presencia y sus acciones. El instrumento del cual se sirve la personalidad es el poder condigno, el cual, según Galbraith, "obtiene la sumisión mediante la promesa o la realidad del castigo" y "se extiende al poder que es ejercitado mediante cualquier forma de acción adversa o mediante su amenaza, incluyendo multas, otras expropiaciones de bienes, represión verbal y condena pública por parte de otros individuos o de la comunidad" (1985, pp. 35-36).

La propiedad es la segunda fuente para ejercer el poder. Así, la tierra en un principio, los recursos financieros después y el capital industrial hoy son capaces de comprar la sumisión de otros. El instrumento que usa la propiedad es el poder compensatorio, definido por Galbraith (1985) como aquel que consigue la sumisión de otras personas mediante la promesa o la materialización de un beneficio y ya no mediante la promesa o materialización de un castigo. Ejemplos del poder compensatorio son los salarios, los premios y los incentivos económicos.

En la actualidad el uso de la propiedad, como fuente que confiere la posibilidad de comprar sumisión, ya no da acceso automático al poder, pues la propiedad aislada de una organización en la cual sea visible, se encuentra desprovista de la capacidad de influir en las decisiones y acciones de otros. Por tanto, la organización emerge como tercera fuente de poder. Ella nombra a un gerente general, quien se encarga de la creación de su superestructura, para lo cual contrata a los gerentes de línea en quienes delega autoridad formal para el ejercicio del poder a través de la denominada cadena de autoridad. Acto seguido, contrata a otros empleados, quienes se encargan directamente de la transformación o elaboración del producto o del ofrecimiento del servicio. Finalmente vincula a personas que se desempeñarán en los organismos asesores y en los de control. El instrumento del que se vale la organización es el poder condicionado, este incluye la persuasión visible explícita en las leyes y reglamentos, como el código laboral y el reglamento interno (Galbraith, 1985). De igual manera abarca la cultura organizacional, la cual es definida por Schein (2004) como el patrón de supuestos básicos compartidos por los miembros de una organización que influye en su forma de percibir, pensar y sentir en relación con los problemas que enfrentan.

Se deduce que la propiedad necesita de la organización para mantenerse viva y, a su vez, esta necesita de sus gerentes para obtener los objetivos económicos, ambientales y sociales. Sin embargo, los gerentes comparten escenario con otros actores o grupos de interés (Freeman, 1994) que compiten por el poder y que demandan su atención: empleados, propietarios, junta directiva, proveedores, clientes, competencia, sindicatos, asociaciones profesionales y público en general. Los gerentes de hoy necesitan, entonces, conocer los motivos que vinculan a los grupos de interés con sus organizaciones, para ejercer influencia mediante el uso de las fuentes e instrumentos de poder a su disposición. Con este conocimiento, pueden balancear las relaciones de poder para responder a las demandas de estos grupos, las cuales incluyen obligaciones morales cuyo cumplimiento o incumplimiento los afecta en gran medida (Tashman y Raelin, 2013). De manera que asumir un rol gerencial abarca un espectro más amplio que el de los aspectos técnicos, ya que requiere también de una ética que guíe el ejercicio del poder.

En este artículo la ética se entiende como aquella parte de la filosofía que reflexiona sobre la moral. Ahora bien, la moral es el conjunto de normas sobre los comportamientos permitidos (buenos) y prohibidos (malos) 
que comparte un grupo humano y que tiene como finalidad regular el comportamiento social. En otras palabras, la ética abarca el análisis y la interpretación de un tipo especial de comportamientos humanos que tienen que ver con lo bueno y lo malo, es decir, con el mundo moral (Arango, 2005).

El poder y la ética tienen una presencia significativa en el devenir de las organizaciones y en sus gerentes. Mediante el uso del poder toman decisiones, emprenden proyectos y asignan recursos. La ética, les ayuda a tomar mejores decisiones a partir de la reflexión sobre las normas que rigen sus comportamientos. Así, poder y ética tienen una estrecha relación, pues el uso de poder genera impactos positivos o negativos sobre las personas y el medioambiente, por lo que es imprescindible ejercerlo con la compañía de la ética.

Si un gerente ha sido educado para hacer un uso ético de su poder, estará preparado para tomar decisiones más responsables y actuar para hacer el bien, no causar daño o remediar el daño causado. Por ejemplo, un gerente ético que se enfrenta a la decisión de despedir el $20 \%$ de su planta de empleados, consideraría, además de los asuntos económicos y operacionales, preguntas tales como ¿es posible tomar otras alternativas?, ¿qué acciones seguir para reducir los impactos negativos de esta decisión? Esto repercutirá positivamente en sus empleados quienes tenderán, a su vez, a tener más alto grado de comportamientos ciudadanos en la organización, tal como se encontró en un estudio realizado por Reiley y Jacobs (2014).

En síntesis, el poder que detentan los gerentes les genera grandes responsabilidades y retos, poniendo a prueba su integridad, lo que exige personas morales en los cargos directivos (Mintzberg, 1983). Un gerente ético hará que los grupos de interés crean en su capacidad para respetar los valores morales que hacen parte de la cultura organizacional, a la vez que logra los objetivos con eficiencia, respondiendo a las expectativas sociales y ambientales que genera la organización en la que se desempeña. Por tanto, una buena parte de los esfuerzos de las universidades debería estar encaminada no solo a formar gerentes con altas calidades técnicas, sino también a desarrollar competencias para el comportamiento moral. Dichas competencias se pueden desarrollar con la ayuda de programas de formación como el que se propone en este artículo. Este se fundamenta en la pedagogía constructivista social y usa un modelo de ética gerencial (Rodríguez, 2008) como fundamento teórico de las actividades que buscan la afirmación de valores morales (Atkins y Parker, 2012), de acuerdo con la premisa de darle voz a los valores para que se constituyan en punto de apoyo en la educación ética gerencial (Arce y Gentile, 2015).

Este artículo se centra en estudiantes de ingeniería por cinco razones. La primera es que ellos se desempeñarán en cargos gerenciales a cualquier nivel (alto, medio, de supervisión), puesto que buscarán el logro de objetivos mediante el trabajo con otras personas y el uso de recursos físicos, financieros, tecnológicos e informacionales, sea como ingenieros civiles, electricistas, electrónicos, industriales, químicos, mecánicos, entre otros. La segunda es que la educación en ingeniería demanda un énfasis ético por las implicaciones sociales de la profesión y por los riesgos que conlleva su práctica (Rodríguez et al., 2010). La tercera es que la ingeniería exige la presencia de profesionales que desplieguen intereses reflexivos, si no se quiere caer en el juego tecnocrático que muchas veces demerita las dimensiones humanas, sociales y ambientales de la profesión (Estrada, 2008). La cuarta es que en la educación universitaria cada día es mayor la tendencia a desarrollar competencias integrales que permitan a los futuros profesionales ser más responsables en su desempeño técnico, social y ambiental (Marines et al., 2014). La quinta es que dos de los autores de este artículo se han desempeñado por más de 20 años como profesores de una facultad de ingeniería y, aunque son conscientes del avance que ha tenido la educación ética en ingeniería (Chung, 2015), saben que aún existe una brecha entre lo que se cree que debe enseñarse y lo que la realidad de la práctica ingenieril requiere. En palabras de Schmidt (2014, p. 986), es necesario "desarrollar una forma de pensar y de aplicar la ética que sea más consistente con las realidades de la práctica de la ingeniería que lo que se encuentra en mucha de la literatura actual."

El presente artículo propone un programa de formación en uso ético del poder gerencial para estudiantes de ingeniería que no reemplaza asignaturas dictadas usualmente en los planes de estudio de ingeniería como ética, ética profesional o deontología, sino que se centra en desarrollar competencias integrales para el adecuado uso del poder que tendrán los ingenieros en su vida laboral. Debido a las restricciones de espacio normales en las publicaciones científicas, este artículo tiene dos limitaciones, una es que no contiene todos los desarrollos teóricos de la investigación y otra es que solo detalla dos de las actividades diseñadas. Quien desee conocer todos los resultados, puede consultar a Rodríguez et al., (2014).

\section{METODOLOGÍA}

Este artículo es resultado de la quinta fase de una línea de investigación en ética gerencial iniciada desde 1997 por miembros del grupo Ethos de la Universidad Nacional de Colombia (Rodríguez et al., 2014). Para esta fase se diseñó una metodología cualitativa que se compone de cinco etapas (ver Figura 1). 


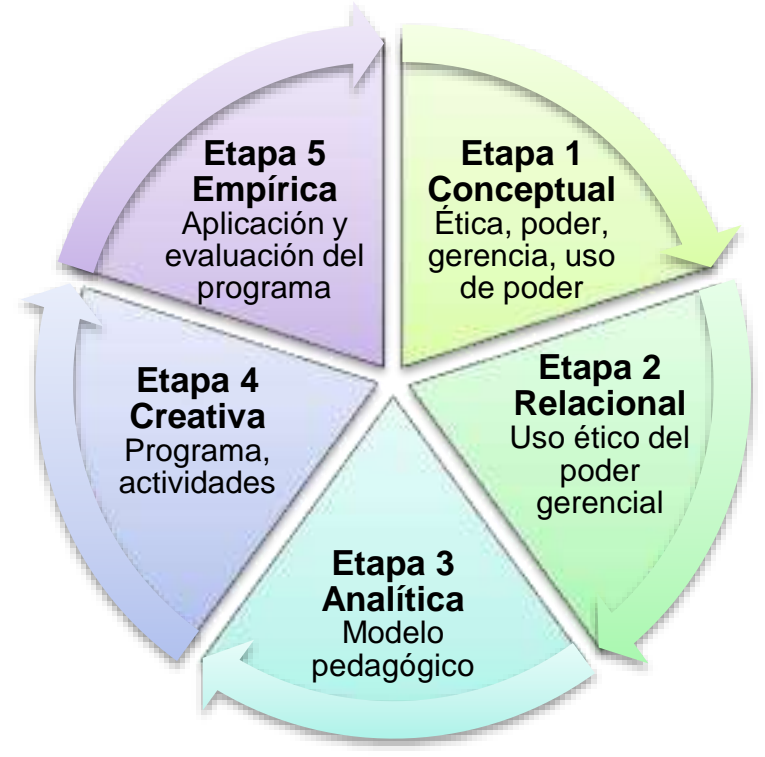

Fig. 1: Modelo metodológico

En la primera etapa - conceptual - se comprendió, interpretó y contextualizó la bibliografía, con el fin de obtener un marco conceptual que da cuenta de los términos centrales del estudio: ética, poder, gerencia y uso de poder. Aquí se abordó el asunto desde la sociología, la administración y la ética organizacional, para tener una mirada interdisciplinaria de estos conceptos. En la segunda etapa - relacional - se recurrió a la técnica del Seminario Alemán (Hoyos, 1988), el cual funciona como estructura de trabajo intelectual crítico en donde los productos se van madurando entre los investigadores hasta lograr los objetivos. En el caso particular, se buscaba obtener un marco teórico que relacionara los conceptos clave de la investigación en torno al uso ético del poder gerencial. En la tercera etapa - analítica -se evaluaron diferentes modelos pedagógicos, con el fin de seleccionar el que permita un adecuado desarrollo de competencias para el uso ético del poder gerencial. En la cuarta etapa - creativa - se hizo acopio de los resultados de las fases anteriores y se diseñaron el programa y las actividades de formación de acuerdo con las últimas tendencias en la ética organizacional (Arce y Gentile, 2015; Fernandes et al., 2014; Freeman et al., 2015; Warren et al., 2014, Nayak, 2015). En la quinta etapa - empírica - se aplicaron las actividades diseñadas a casos concretos, de tal manera que se obtuviera retroalimentación y se pudiera mejorar la propuesta.

Uno de los casos fue la formación en uso ético del poder gerencial para estudiantes de ingeniería. Desde el 2010 se han aplicado las actividades diseñadas en la investigación en la Cátedra Ethos. Dicha Cátedra se ofrece como electiva en la Universidad Nacional de Colombia - Sede Manizales y convoca a estudiantes de las ingenierías que ofrece la Sede. La asignatura se dicta en sesiones semanales de tres horas, su cupo es de 30 estudiantes y tiene 3 créditos. Para la elaboración del presente artículo, al finalizar el segundo semestre de 2015 se hizo una encuesta a los estudiantes inscritos en la Cátedra Ethos. Asimismo, se hicieron entrevistas a cuatro ingenieros con experiencia laboral que cursaron dicha Cátedra en semestres anteriores. Todo esto con el fin de evaluar los efectos de la propuesta. Las respuestas fueron analizadas con la ayuda del software cualitativo ATLAS.ti. A todos ellos se les hicieron las siguientes dos preguntas:

1. ¿Considera que las actividades realizadas durante la Cátedra Ethos lograron incrementar su sensibilidad frente al uso del poder gerencial que usted ejercerá como profesional?, ¿por qué?

2. ¿Cuáles competencias para el uso ético del poder gerencial cree que logró desarrollar a partir de las actividades realizadas en la Cátedra Ethos? Por favor, para cada competencia explique por qué.

\section{REFERENTE TEÓRICO}

La estructura teórica del programa de formación en uso ético del poder gerencial se muestra en la Figura 2. Esta estructura es resultado de las etapas 1 y 2 de la presente investigación. Asimismo, tuvo en cuenta los resultados de las cuatro fases anteriores de la línea en ética gerencial reseñada en la metodología (Rodríguez; 2008). A continuación se hará una breve discusión sobre moral, ética, valores morales y ética gerencial. Ya en la introducción se discutió sobre el poder, el uso de poder en las organizaciones, la gerencia y el uso de poder en la gerencia. Por restricciones de espació no se incluye una discusión sobre ética organizacional, valores morales gerenciales y responsabilidad gerencial, aunque son temas que se desarrollaron en la investigación y que se estudian en el programa. 


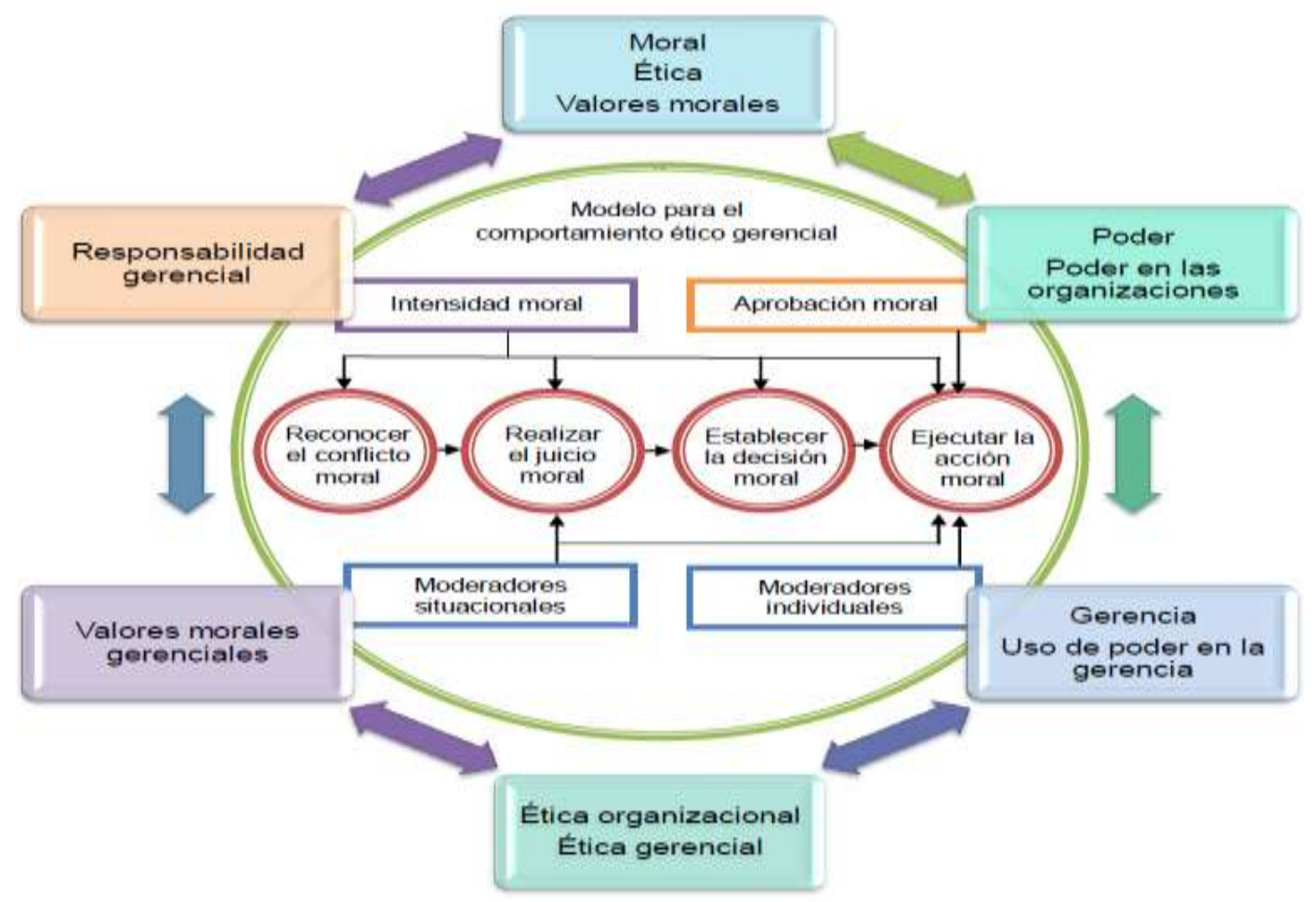

Fig. 2: Estructura teórica del programa

\section{Moral, ética y valores morales}

La moral se refiere a un conjunto de normas sobre el comportamiento permitido que rige a una determinada colectividad o sociedad. Por ello no existe una moral única, sino diferentes morales de acuerdo con el grupo humano o con el período histórico en el que se esté viviendo (Sánchez, 2005). Esto podría ser confuso, ya que es posible que dos personas hagan un juicio diferente sobre un mismo hecho. Por ejemplo, la moral musulmana acepta que un hombre pueda tener hasta cuatro esposas, mientras que la moral católica solo permite una. Sin embargo, si se analiza esta diferencia se explicita el propósito de ambas morales para regular las relaciones maritales. Así, no está permitido a un musulmán tener más de cuatro esposas, como no está permitido a un católico tener más de una. Esta diversidad de morales no debería ser problemático dado que "un concepto de moralidad que no deja abierta la posibilidad de concepciones variadas de lo moral tiene que parecer hoy inaceptable" (Tugendhat, 1997, p. 54).

No se trata de un relativismo moral, sino de la posibilidad de encontrar mínimos comunes entre diversas morales, de tal manera que se posibiliten unos acuerdos intersubjetivos e interculturales. Empero, esos acuerdos requieren que se acepte que los juicios morales son objetivos y contextuales a la vez (Arango, 2005). Objetivos porque la moralidad no depende de gustos o preferencias, sino que hay consensos, tácitos o explícitos, sobre lo bueno y lo malo del comportamiento (p.e. matar es malo, robar es malo, engañar es malo, ayudar a otros en necesidad es bueno, respetar a los demás es bueno). Contextuales porque los juicios no solo tienen en cuenta los acuerdos sobre lo bueno o malo, sino también las circunstancias y los motivos que rodean determinados comportamientos.

Ahora bien, la ética se entiende como la reflexión filosófica acerca de la moral y, en consecuencia, es una parte de la filosofía, como lo son la metafísica, la epistemología o la estética (Tugendhat, 1997). Aquí es importante tener en cuenta que la ética no se encarga de establecer lo que es bueno o malo, sino de estudiar por qué determinados comportamientos son catalogados como buenos y otros como malos. Por lo tanto, la ética no dice cómo actuar, sino que busca los criterios que justifican actuar de un modo o de otro. Asimismo, busca responder a preguntas relacionadas con el qué, el cómo, el por qué y el para qué de las realidades morales, buscando razones que expliquen para qué sirve la moralidad.

Los valores son estudiados por la axiología y, en particular, los valores morales son estudiados por la ética, entendiéndolos como aquellas cualidades que no son atribuibles a objetos particulares, sino a la conducta humana, que se imponen como pautas de acción y pueden o no estar alineados con los deseos individuales, pero motivan su realización como medio para ser aceptado socialmente. El valor moral trasciende al ser físico y llega a la esencia del ser humano haciéndolo más pleno, más persona, reflejando sus comportamientos en las relaciones sociales que lleva diariamente (Cortina, 2003). 
Un valor es moral si su aplicación tiene consecuencias positivas que afectan a otras personas o grupos sociales (Sánchez, 2005), de lo contrario, sería un antivalor. Ahora bien, solucionar un conflicto entre un valor y un antivalor es sencillo: todos preferimos la honestidad a la deshonestidad, la responsabilidad a la irresponsabilidad o el respeto al irrespeto; pero resolver un conflicto entre uno o varios valores es complejo, puesto que "los conflictos morales profundos y dramáticos surgen entre dos valores positivos, entre dos obligaciones morales que atender" (Frondizi, 2001, p. 221). Por lo tanto y dado que "en la vida diaria rara vez se produce un conflicto entre dos valores aislados, sino entre dos constelaciones de valores que adquieren significado distinto según el fondo axiológico de cada cual" (Frondizi, 2001, p. 226), se puede afirmar que para tomar una decisión adecuada es de singular ayuda la ética.

\section{Ética gerencial}

Los tiempos modernos se caracterizan por el predominio de las organizaciones, las cuales están inmersas en complejas relaciones de poder, dentro de las que el gerente ocupa un rol de primera línea. Por ello, Cortina (1998) afirma que así como en tiempos pasados el explorador, guerrero, santo, maestro de escuela, militar o ingeniero, entre otros, marcaron el carácter de su época, en esta sociedad managerial el gerente es el personaje emblemático: "El suyo es ya un liderazgo social y por ello se espera de él una conducta ejemplar. Esto significa que no debe comportarse como sabemos que nos comportamos todos; se espera que proceda como sabemos que debemos proceder nosotros. Cuanto más escépticos nos hemos vuelto con respecto a la conducta de otros líderes — políticos, predicadores o abogados-, más virtud esperamos de los nuevos directivos." (Cortina, 1996, p. 95).

En este sentido, Ferrell y Fraedrich (1994) proponen una variable denominada los otros importantes, la cual se refiere a los superiores, colegas y subordinados que son importantes para una persona y que influencian sus decisiones éticas. Estos autores encontraron que los gerentes tienen una gran influencia en la toma de decisiones, pues ellos pueden ofrecer ejemplos de conducta, tienen el poder para motivar a los empleados y pueden recompensarlos o castigarlos: "Si los gerentes y colegas proveen modelos positivos, los otros importantes pueden convertirse en una fuerza que ayuda a los individuos a tomar mejores decisiones éticas. Pero cuando existe un conflicto severo de roles y presión para cometer acciones no éticas, el comportamiento no ético en la empresa puede crecer." (Ferrell y Fraedrich, 1994, p. 146).

De aquí la necesidad de la ética gerencial, pues su estudio podrá dar claves para proponer diferentes medios para contribuir al mejoramiento de la labor de los gerentes, partiendo de una comprensión de los motivos subyacentes que llevan a los gerentes a comportarse de un modo o de otro. Aquí, entra a colación el modelo para el comportamiento ético gerencial (ver Figura 2) que se explica en los siguientes párrafos.

El eje del modelo es el proceso para la toma de decisiones morales de Rest (1986), el cual se compone de cuatro etapas: 1) reconocimiento del conflicto moral; 2) reflexión usando el razonamiento moral; 3) decisión de actuar de acuerdo con la reflexión moral y; 4) comportamiento moral. Otro de los componentes del modelo es la intensidad moral (Jones, 1991), la cual se compone de seis variables: 1) Magnitud de consecuencias, definida como el grado de beneficios o daños que ocasiona la acción moral. 2) Consenso social, explicado como el grado de acuerdo social sobre lo adecuado o inadecuado de la acción. 3) Probabilidad de efecto, entendida como la posibilidad de que un acto pueda ocurrir y causar beneficios o daños. 4) Inmediatez temporal, definida como el periodo de tiempo entre la toma de la decisión y sus consecuencias. 5) Proximidad personal, explicada como la cercanía que se tiene con las personas que sufrirán las consecuencias de la acción. 6) Concentración de efectos, referente a si la acción recae sobre un pequeño grupo de personas (alta concentración) o sobre una gran cantidad de ellas (baja concentración). Jones propone que entre más alta la intensidad, más alta la frecuencia de comportamientos morales.

La aprobación moral, planteada por Jones y Ryan (1997, 1998), ayuda al entendimiento del paso de la decisión moral al comportamiento moral. Aquí se asume que el deseo de aprobación moral del grupo referente y el nivel de responsabilidad moral que la persona anticipa que le será atribuido por su organización, afectan los vínculos entre el pensamiento y las acciones morales. Los autores definen la aprobación moral como el deseo de las personas de ser catalogadas como éticas por sí mismas, su grupo de referencia en la organización y otras personas relacionadas. Así, entre más alta la aprobación moral, más alta la probabilidad de actuar bajo la decisión moral que se tomó.

Los moderadores situacionales e individuales planteados por Treviño (1986), se explican mediante un modelo que contiene variables situacionales como el contexto de trabajo (refuerzos, presiones), cultura organizacional (héroes, estructura normativa, responsabilidad por las consecuencias, obediencia a la autoridad) y características del trabajo (asunción de roles, resolución de conflictos morales). Asimismo, contiene variables individuales como el ego (fortaleza de la convicción en las habilidades de autorregulación), el campo de dependencia (grado de subordinación a fuerzas sociales externas), el punto 
de control (grado en que las personas ejercen control sobre su propia vida) y la etapa de desarrollo moral según la Teoría del Desarrollo Moral Cognitivo (CMD) de Kohlberg (1976). Esta teoría ha sido ampliamente aceptada por investigadores en ética gerencial, aunque también tiene un gran número de críticos (Maclagan, 1996); ella plantea que desde la infancia hasta la adultez, las personas avanzan en un proceso de desarrollo moral a través de las siguientes etapas: 1) moralidad heterónoma, 2) moralidad individualista e instrumental, 3) moralidad interpersonal y normativa, 4) moralidad del sistema social, 5) moralidad de los derechos humanos y el bienestar social y 6) moralidad de los principios universales.

Las facultades de ingeniería pueden contribuir al desarrollo de competencias para el uso ético del poder a partir de una formación que ayude a los estudiantes a avanzar en su etapa de CMD, a fortalecer su autonomía, a incrementar su capacidad de discriminación de lo bueno y lo malo y a defender sus convicciones morales. Todo lo anterior con una fuerte fundamentación en valores morales como el diálogo, la honestidad, la solidaridad, la justicia, el respeto y la responsabilidad. Asimismo, pueden promover una cultura organizacional ética con el apoyo de refuerzos al comportamiento ético, buen ejemplo por parte de los profesores y directivos, reglamentos estudiantiles con responsabilidades claras, canales de comunicación que promuevan el diálogo, entre otros moderadores situacionales. En todo caso, se debe tener en cuenta que los estudiantes vienen con predisposiciones individuales que influencian su comportamiento, pero también que los factores situacionales ejercen un gran impacto en ellos.

\section{PROPUESTA}

Como resultado de las etapas 3 y 4 de la investigación, se diseñó un programa que puede ser adaptado a las características de la universidad que decida aplicarlo y a los lineamientos de las ingenierías donde se inscriba. El programa se enmarca en la pedagogía constructivista social (Flórez, 2005). Se escogió esta pedagogía porque ella permite estudiar un fenómeno dado dentro de su contexto y desarrollar una mejor comprensión de las causas que lo originan. Su meta se basa en el desarrollo intelectual del ser humano de forma progresiva, por medio del análisis crítico y la reflexión, teniendo en cuenta las necesidades y condiciones particulares del aprendiz. Así pues, la premisa del constructivismo pedagógico, "es que el conocimiento es construido por el educando, y no impartido por el maestro. Sus instrumentos son los que amplían la capacidad de los alumnos para explorar y experimentar" (De Moura, 1998: 31).

Una de las bondades del constructivismo radica en el reconocimiento que se hace de la participación de las personas en un proceso de construcción que las lleva a modificar su estructura mental y alcanzar un mayor nivel de aprehensión. El constructivismo reconoce el desarrollo integral del ser humano dentro del proceso formativo y toma en cuenta las funciones emotivas y motrices, además de las cognitivas, de tal forma que al considerar estos aspectos se puede lograr el máximo desarrollo del potencial humano (Maturana y VerdenZöller, 1993). Por esto, la metodología empleada en las actividades del programa se basa en el aprendizaje activo, que hace parte de la construcción colectiva de los participantes (Fernandes et al., 2014). Esta metodología tiene como consigna el trabajo en equipo, ya que la disposición física y mental de los involucrados hace que cada tarea se convierta en reto, responsabilidad y enseñanza.

El objetivo del programa es desarrollar en los estudiantes competencias para la reflexión ética, la toma de decisiones éticas y la solución de conflictos éticos relacionados con el uso del poder gerencial. Al terminar el programa, los estudiantes podrán: (i) conceptualizar acerca de lo que significa: la moral, la ética, los valores morales, el poder, la gerencia, el uso de poder en la gerencia, la ética organizacional, la ética gerencial, los valores morales gerenciales y la responsabilidad gerencial; (ii) analizar casos de la vida real que ilustren conflictos éticos relacionados con el uso del poder gerencial; (iii) identificar sus responsabilidades individuales, sociales y políticas como ingenieros y como gerentes; (iv) tomar decisiones éticas.

Se entiende por competencia "un saber hacer frente a una tarea específica, la cual se hace evidente cuando el sujeto entra en contacto con ella. Esta competencia supone conocimientos, saberes y habilidades que emergen en la interacción que se establece entre el individuo y la tarea y que no siempre están de antemano" (Corpoeducación, 2003, pp. 3-4). Rodríguez (2008) señala la necesidad de desarrollar competencias integrales en los gerentes, las cuales no solo involucran el saber y el saber hacer, sino también el ser. Las competencias del ser se componen de actitudes, comportamientos y valores. Son éstos últimos a los que se apunta en la propuesta, específicamente a los valores morales.

En la Tabla 1 se presenta el contenido general del programa. La primera sesión corresponde a una introducción al programa y al grupo en formación. Esta primera sesión sirve para mostrar las estrategias pedagógicas que se utilizarán a lo largo del programa y los compromisos que adquieren los integrantes del grupo. En las siguientes sesiones, se van abordando los temas centrales de la asignatura, teniendo en cuenta que cada sesión magistral debe ser seguida de una sesión de actividades. Los estudiantes deberán hacer unas lecturas obligatorias que les permitirán construir una conceptualización propia de los temas 
centrales del curso, producto de sus interacciones teóricas y vivenciales. En las sesiones teóricas y en las actividades se deben incluir asuntos relacionados con las obligaciones morales y legales de los ingenieros, tal como lo plantean Warren et al. (2014). Estos autores proponen una formación ética integral que tenga un enfoque dual - valorativo y normativo - tanto en los contenidos como en las actividades a realizar.

Tabla 1: Contenido general del programa

\begin{tabular}{|c|l|l|}
\hline Sesión & Actividad & Lecturas obligatorias \\
\hline 1 & Introducción, compromisos & \\
\hline 2 & Moral, ética, valores morales & Rachels (2007, cap. 1) \\
\hline 3 & Actividad: el juego del BAFA - BAFA (Rodríguez, 2008) & Rachels (2007, cap. 2) \\
\hline 4 & Poder, poder en las organizaciones & Cantor (2014) \\
\hline 5 & Actividad: La organización (Vásquez, 2014) & Rachels (2007, cap. 3) \\
\hline 6 & Gerencia, uso de poder en la gerencia & Pantoja (2014) \\
\hline 7 & Actividad: Un enemigo del pueblo & Ibsen (2007) \\
\hline 8 & Ética organizacional & Rodríguez (2014) \\
\hline 9 & Actividad: En los zapatos del otro (Vásquez, 2014) & Rachels (2007, cap. 4) \\
\hline 10 & Ética gerencial & Rodríguez (2014) \\
\hline 11 & Caso de estudio: Whistleblowing en la Universidad Tayrona (Vásquez, 2014) & Rachels (2007, cap. 5) \\
\hline 12 & Responsabilidad gerencial & Tello y Rodríguez (2014) \\
\hline 13 & Actividad: El desastre de Chernóbil & Rachels (2007, cap. 6) \\
\hline
\end{tabular}

\section{Actividades}

Las actividades pretenden llevar a que los participantes interioricen los valores y normas que rigen el ejercicio de la ingeniería de diferentes formas (teórica, experiencial e introspectiva). Al respecto, se recomienda que las actividades involucren lo que Nayak (2015) denomina asuntos trágicos, refiriéndose a situaciones en que una serie de eventos conducen a consecuencias nefastas; esto presenta una oportunidad para ganar sabiduría personal y laboral (ver El desastre de Chernóbil). Asimismo, se sugiere incluir actividades relacionadas con la literatura, el cine o el teatro, que permitan a los participantes conectarse consigo mismos, con los otros y con la complejidad humana (Freeman et al., 2015) de una forma diferente a la que usualmente se utiliza en los cursos de formación gerencial (ver actividad Un enemigo del pueblo). En general, Warren et al. (2014) afirman que las actividades serán más efectivas si se hacen cara a cara, si involucran a los participantes en asuntos que han ocurrido o pueden ocurrirles y si generan conclusiones que parten de una reflexión colectiva entre pares. A manera de ejemplo, a continuación se muestran dos de las actividades mencionadas en la Tabla 1.

\section{El desastre de Chernóbil}

Materiales: copias del prólogo de la obra Voces de Chernóbil (Aleksiévich, 2015), copia del documental El desastre de Chernóbil (Discovery Channel, 2012). Duración: 150 minutos (20 para la lectura del prólogo, 15 para la discusión inicial, 95 para la proyección de la película, 20 para la discusión final). Desarrollo: se dará inicio a la sesión haciendo una lectura del prólogo del libro Voces de Chernóbil (Aleksiévich, 2015), luego se hará una discusión de las impresiones que dejó en los estudiantes el prólogo. A continuación se hará una breve reseña del documental El desastre de Chernóbil (Discovery Channel, 2012) y se les solicitará que mientras ven la película piensen en qué decisiones hubieran tomado en el caso de ser el ingeniero a cargo del experimento previo al accidente de Chernóbil, o si los hubieran nombrado en el equipo de ingenieros nucleares que debió intervenir inmediatamente después del desastre, o si estuviesen dentro del comité encargado de la liquidación del accidente que se llevó a cabo en los meses posteriores a la catástrofe.

Discusión: una vez terminado el documental se hacen preguntas tales como: ¿cuáles fallos cometieron los ingenieros a cargo de la construcción de la planta nuclear?, ¿los encargados de continuar con la operación de la planta?, ¿cuáles valores están en juego?, ¿cómo hubieran actuado ustedes si hubieran sido el ingeniero a cargo del experimento que desató el desastre?, ¿cómo asumieron las autoridades soviéticas sus responsabilidades frente a la catástrofe? Por favor, analicen las decisiones que tomó Valery Legásov durante los dos años siguientes a la catástrofe, ¿consideran un acto de responsabilidad su suicidio?

\section{Un enemigo del pueblo}

Materiales: papel y lápiz para todos, copia digital de la obra Un enemigo del pueblo (Ibsen, 2007). Duración: 150 minutos (10 para la introducción, 40 para la preparación de la obra, 60 para las presentaciones de los grupos y 40 minutos para la discusión). Desarrollo: antes de la sesión presencial se pedirá a los participantes que lean la obra de teatro Un enemigo del pueblo de lbsen (2007), en la que se narra la historia del Dr. Stockmann, quien denunció que el balneario de su pueblo se surtía de aguas altamente 
perjudiciales para la salud de los bañistas y como consecuencia perdió su trabajo, sus hijos fueron despedidos de la escuela y del trabajo, su casa fue apedreada y él mismo fue catalogado como un enemigo del pueblo. Una vez en la sesión, se organizan grupos de seis estudiantes y se les pide que preparen y presenten una obra de teatro de 10 minutos.

Discusión: en un primer momento se hacen las siguientes preguntas: ¿Cómo se sintieron desempeñando los papeles en la obra?, ¿cuáles valores están en juego?, ¿qué hubieran hecho ustedes en lugar del Dr. Stockman?, ¿por qué?, ¿a quiénes les debía mayor lealtad el Dr. Stockman, a los habitantes de su pueblo o a los bañistas que lo visitaban? En un segundo momento se les pide que se reúnan de nuevo en sus grupos y que respondan a la siguiente pregunta ¿cómo podrían relacionar esta actividad con el trabajo que van a desarrollar como ingenieros?, por favor den algunos ejemplos. Finalmente, se pide a cada grupo que presente las respuestas en plenaria y se abre de nuevo la discusión.

\section{EVALUACIÓN DE LA PROPUESTA}

Uno de los resultados de la etapa 5 de la investigación fue la evaluación del programa presentado en la Tabla 1. Al finalizar el segundo semestre de 2015 se hizo una encuesta a los estudiantes que estaban cursando la Cátedra Ethos, la cual fue respondida por la totalidad de ellos (30). De igual manera, y con el fin de triangular la información, se hicieron cuatro entrevistas semiestructuradas (con las mismas dos preguntas) a ingenieros que cursaron la Cátedra Ethos en semestres anteriores. A continuación se presentan los principales resultados y su análisis.

1. ¿Considera que las actividades realizadas durante la Cátedra Ethos lograron incrementar su sensibilidad frente al uso del poder gerencial que usted ejercerá como profesional?, ¿por qué?

Todos los encuestados y entrevistados respondieron afirmativamente a esta pregunta. Las razones que dieron para su respuesta se agruparon, con la ayuda del ATLAS.ti, en tres familias: (i) Les permitió desarrollar habilidades; (ii) Lograron afianzar valores morales; and (iii) Les aportaron conocimientos sobre el uso ético del poder gerencial

Se puede deducir del análisis de las respuestas que el programa de formación que se propone en este artículo y que se aplicó en la Cátedra Ethos, logró incrementar la sensibilidad de los estudiantes de ingeniería frente al uso del poder gerencial, permitiéndoles desarrollar habilidades, afianzar valores morales y adquirir conocimientos. En palabras de un estudiante de décimo semestre de Ingeniería Química: "Siempre pensé que el conocimiento era poder o un alto cargo como un gerente era poder innato sin importar cómo lo usara, pero la Cátedra Ethos me ha otorgado un nuevo punto de vista, me ha dado herramientas para dimensionar todo lo que el término poder en la gerencia implica. Me llevo de la Cátedra conceptos valiosos que de seguro honraré aplicarlos, quiero ser un líder que sepa hacia dónde quiere ir, que trate a su organización con respeto, honestidad y justicia, quiero explorar todos los mecanismos que conduzcan a mis objetivos. Me asusto porque me iba a graduar sin saberlo, ¿cuántos colegas míos van a graduarse sin saberlo?".

Un ingeniero electrónico que cursó la Cátedra Ethos en el primer semestre de 2013 y que tiene dos años de experiencia laboral, corroboró lo afirmado en el párrafo anterior: Como producto de la Cátedra Ethos, tres valores quedaron muy gravados en mi mente. La honestidad, la responsabilidad y el respeto. Esto hace que yo sea reconocido en la organización como una persona que cumple con lo que es y por eso confían en mí. Esto también hace que me elijan para desempeñar tareas delicadas, veo que mis superiores tienen una muy buena opinión de mí por ser una persona confiable.

2. ¿Cuáles competencias para el uso ético del poder gerencial cree que logró desarrollar a partir de las actividades realizadas en la Cátedra Ethos? Por favor, para cada competencia explique por qué.

Las respuestas de los encuestados se agruparon en tres familias (competencias del saber, competencias del ser y competencias del hacer). Con la ayuda del ATLAS.ti se construyó una red que permite visualizar los elementos asociados a cada competencia (ver Figura 3). Se puede observar que cada elemento contiene dos números entre paréntesis, el primer número se refiere a la cantidad de encuestados que mencionaron el elemento en sus respuestas. Las competencias del ser, expresadas como la capacidad de vivir valores morales, fueron las más mencionadas por los encuestados, destacándose el respeto y la responsabilidad. El diálogo también se destacó por pertenecer a las competencias del ser (como valor moral) y a las del hacer (como habilidad de comunicarse con otros). Al respecto, una estudiante de noveno semestre de Ingeniería Industrial afirmó: "Me pareció significativo aprender la importancia del ser. En la gerencia contemporánea no es suficiente con saber y con hacer, este es un aspecto muy motivante ya que 
le da la suficiente relevancia al crecimiento personal en las organizaciones. También considero que fortalecí la habilidad de aplicación de valores, ya que comprendí que las personas son el activo intangible de las organizaciones y que su formación en valores puede ser vital para la mejora del clima organizacional.

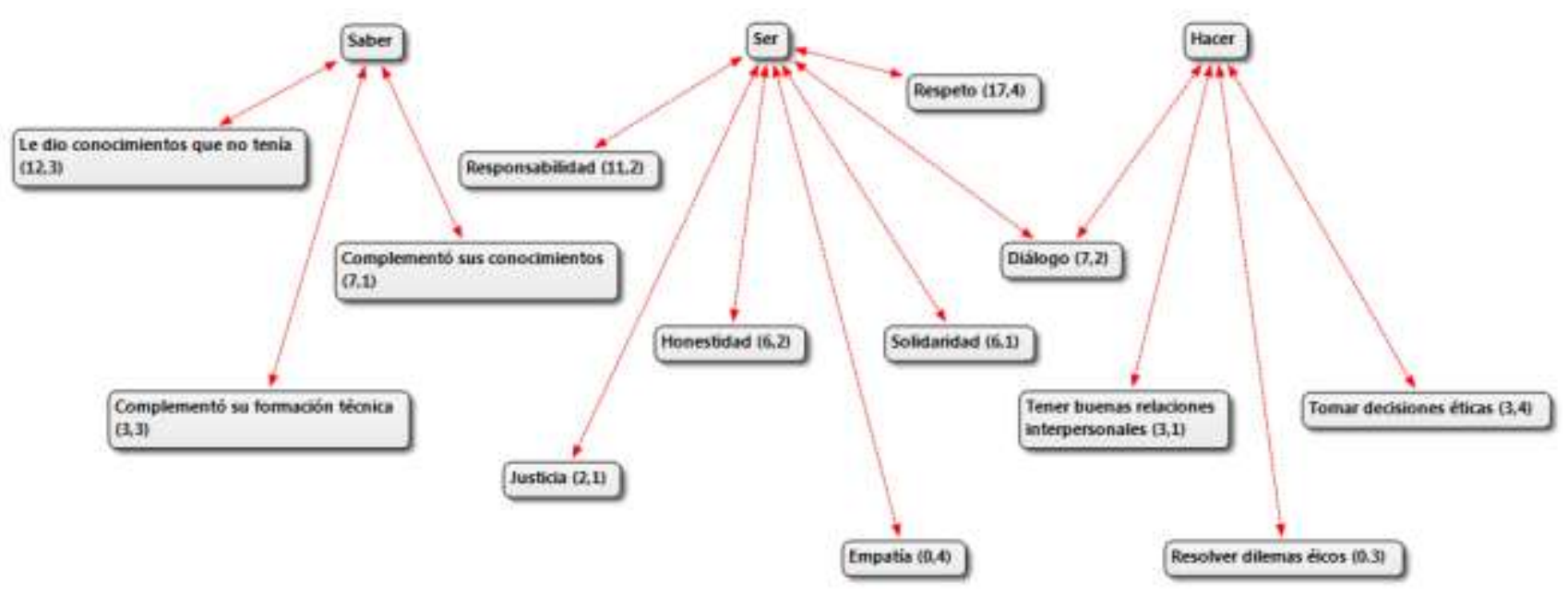

Fig. 3.: Competencias desarrolladas a partir de las actividades de la Cátedra Ethos

Para analizar las respuestas de los entrevistados se utilizaron las mismas familias y elementos de los encuestados. La Figura 3 muestra dichas relaciones y mediante el segundo número que aparece entre paréntesis se muestra la cantidad de entrevistados que mencionaron el elemento en sus respuestas. Se puede observar que, tal como respondieron los encuestados, el énfasis de la formación se da en las competencias del ser y el valor más destacado es el del respeto. Como elemento diferenciador, aparece dentro de las competencias del ser la empatía o capacidad de ponerse en el lugar del otro, aspecto fundamental para la reflexión ética. Igualmente, en las competencias del hacer se menciona la capacidad para resolver dilemas éticos y se le da gran importancia a la habilidad para tomar decisiones éticas. Estas respuestas, al provenir de ingenieros con experiencia laboral, corroboran que el programa propuesto puede contribuir a desarrollar competencias integrales relacionadas con el uso ético del poder gerencial.

Vale anotar que a los entrevistados se les solicitó que hicieran sugerencias para mejorar la Cátedra Ethos. Al respecto, todos coincidieron en la necesidad de realizar un mayor número de actividades de larga duración, pues fueron éstas las que más se quedaron en su memoria. Dos de ellos fueron insistentes en que cursos como éste deberían ser obligatorios en la formación de los ingenieros y uno más sugirió que deberían darse unas tres veces durante los estudios, pues así se reforzarían los aprendizajes. Es pertinente culminar esta sección con una cita de un ingeniera civil que se encuentra trabajando en una empresa de la construcción de vivienda: El haber cursado la Cátedra Ethos me sirvió para adquirir conocimientos diferentes a los técnicos de mi profesión, conocimientos sobre la ética y la moral. También me ayudó a afianzar valores como el respeto, el diálogo y la solidaridad. Eso unido a mi formación académica y a otros valores que ya tenía muy desarrollados como la honestidad y la responsabilidad han contribuido a que marque la diferencia en sentido positivo en las organizaciones donde he trabajado.

\section{CONCLUSIONES}

A partir de los resultados se pueden indicar las siguientes conclusiones: 1) Un programa de formación en uso ético del poder para estudiantes de ingeniería debe buscar el desarrollo de competencias integrales. 2) La pedagogía constructivista social facilita el desarrollo de dichas competencias, a través de actividades relacionadas con casos de estudio, asuntos trágicos, literatura y vivencias personales. 3) El programa de formación propuesto puede desarrollar competencias relacionadas con el saber, el hacer y, especialmente, el ser. 4) El programa puede ser replicado en otras universidades que deseen formar a sus estudiantes de ingeniería en uso ético del poder gerencial, con énfasis en el afianzamiento de valores morales como el respeto, la responsabilidad, el diálogo, la honestidad y la solidaridad; el desarrollo de habilidades para la solución de dilemas éticos y la toma de decisiones éticas; y el aprendizaje de conocimientos, que complementen su formación técnica, sobre ética, poder, gerencia y uso de poder en la gerencia.

\section{REFERENCIAS}

Aleksiévich, S., Voces de Chernóbil, Penguin Random House, Barcelona, España (2015)

Arango, P.R., Introducción a la filosofía moral, Editorial Universidad de Caldas, Manizales, Colombia (2005) 
Arce, D.G. y M.C. Gentile, Giving Voice to Values as a Leverage Point in Business Ethics Education, Journal of Business Ethics, 131(3), 535-542 (2015)

Atkins, P.W.B. y S.K. Parker, Understanding Individual Compassion in Organizations: The Role of Appraisals and Psychological Flexibility, Academy of Management Review, 37 (4), 524-546 (2012)

Cantor, F., Poder, en Formación gerencial en uso de poder: un enfoque ético, por M.P. Rodríguez, F. Cantor, M.A. Pantoja, J. Toro y A.F. Vásquez, pp 19-41, Editorial UN, Bogotá, Colombia (2014)

Chung, C., Comparison of Cross Culture Engineering EthicsTraining Using the Simulator for Engineering Ethics Education, doi: 10.1007/s11948-014-9542-z, Science and Engineering Ethics, 21, 471-478 (2015)

Corpoeducación, Competencias laborales: base para mejorar la empleabilidad de las personas, Ministerio de Educación Nacional de la República de Colombia, Bogotá, Colombia (2003)

Cortina, A., Ética de la empresa. Claves para una nueva cultura empresarial, Trotta, Madrid, España (1996)

Cortina, A., Business ethics in the catholic value system: The spanish case, en Ethics in international management por B.N. Kumar y H. Steinmann, pp. 401-418, Walter de Gruyter, Berlín, Alemania (1998)

Cortina, A., El mundo de los valores, Editorial El Búho, Bogotá, Colombia (2003)

De Moura, C., La educación en la era de la informática, BID, Washington D.C., USA (1998)

Discovery Channel, El desastre de Chernobyl, www.youtube.com/watch?v=kJMPERF41P8 (2012)

Estrada, E., La enseñanza de la ingeniería en un mundo globalizado, http://www.educacioneningenieria.org/ index.php/edi/article/view/154/133, ISSN 1900-8260, Revista Educación en Ingeniería, 5, 74-78 (2008)

Fayol, H., General and Industrial Management, Pitman, Londres, Reino Unido (1949)

Fernandes, I.M., D.M. Pires y R.M. Villamañán, Educación Científica con Enfoque Ciencia-TecnologíaSociedad-Ambiente. Construcción de un Instrumento de Análisis de las Directrices Curriculares, doi: 10.4067/S0718-50062014000500004, Formación Universitaria, 7(5), 23-32 (2014)

Ferrell, O.C. y J. Fraedrich, Business Ethics, $2^{a}$ edición, Houghton Mifflin, Boston, USA (1994)

Flórez, R., Pedagogía del conocimiento, 2ª edición, McGraw Hill, Bogotá, Colombia (2005)

Freeman, R.E., A stakeholder theory of the modern corporation, en Ethical Theory and Business, $5^{\mathrm{a}}$ edición, por T. Beauchamp y N. Bowie, pp. 38-48, Prentice Hall, Englewood Cliffs, USA (1994)

Freeman, R.E., L. Dunham, G. Fairchild y B. Parmar, Leveraging the Creative Arts y Business Ethics Teaching, Journal of Business Ethics, 131(3), 519-526 (2015)

Frondizi, R., ¿Qué son los valores? Fondo de cultura Económica, México D.F., México (2001)

Galbraith, J.K., La Anatomía del Poder, 2ª edición, Plaza \& Janes Editores, Barcelona, España (1985)

Hoyos, J., El seminario en la experiencia docente de la Facultad de Filosofía de la Universidad Javeriana, Revista Universitas Philosophica, 10, 44-47 (1988)

Ibsen, H., Un enemigo del pueblo, Losada, Buenos Aires, Argentina, (2007)

Jones, T.M., Ethical decision making by individuals in organisations: An issue-contingent model, Academy of Management Review, 16(2), 366 - 395 (1991)

Jones, T.M. y L.V. Ryan, The link between ethical judgement and action in organizations: $A$ moral approbation approach, Organization Science, 8(6), 663-680 (1997)

Jones, T.M. y L.V. Ryan, The effect of organizational forces on individual morality: Judgement, moral approbation, and behavior, Business Ethics Quarterly, 8(3), $431-445$ (1998)

Kemp, L.J., Modern to postmodern management: developments in scientific management, Journal of Management History, 19(3), 345-361 (2013)

Kohlberg, L., Moral stages and moralization. The cognitive-developmental approach, en Moral Development and Behavior, por T. Likona, pp 170-205, Holt, Rinehart \& Winston, Nueva York, USA (1976)

Maclagan, P., The Organisational Context for Moral Development, Questions of Power and Access, Journal of Business Ethics, 15(6), 645-654 (1996) 
Marines, M.S., N.G. Heredia, L.E. Solis y D.A. Mena, Taller Multidisciplinario para el Desarrollo de Competencias de Comunicación Lingüística de la Investigación, doi: 10.4067/S0718-50062014000200006, Formación Universitaria, 7(2), 41-50 (2014)

Maturana, H. y G. Verden-Zöller, Amor y juego: fundamentos olvidados de lo humano, Editorial Instituto de Terapia Cognitiva, Santiago de Chile, Chile (1993)

Mintzberg, H., El trabajo del directivo: Folclore y realidad, en Liderazgo por Harvard Business Review, pp 138, Deusto, Buenos Aires, Argentina (2004)

Mintzberg, H., Power in and Around Organizations, Prentice Hall, Englewood Cliffs, USA (1983)

Nayak, A., Wisdom and the Tragic Question: Moral Learning and Emotional Perception in Leadership and Organisations, doi: 10.1007/s10551-015-2540-5, Journal of Business Ethics, 1-13 (2015)

Oswick, C., P. Fleming y G. Hanlon, From Borrowing to Blending: Rethinking the Processes of Organizational Theory Building, Academy of Management Review, 36(2), 318-337 (2011)

Pantoja, M.A., Uso de poder en la gerencia, en Formación gerencial en uso de poder: un enfoque ético, por M.P. Rodríguez, F. Cantor, M.A. Pantoja, J. Toro y A.F. Vásquez, pp 43-77, Editorial UN, Bogotá, Colombia (2014)

Pearson, A.E., Seis tareas básicas de los gerentes generales, en Oficio y arte de la gerencia, Volumen 1, pp 1-19, Norma, Bogotá, Colombia (1995)

Rachels, J., Introducción a la filosofía moral, Fondo de Cultura Económica, México D.F., México (2007)

Reiley, P.J. y R.R. Jacobs, Ethics Matter: Moderating Leaders' Power Use and Followers' Citizenship Behaviors, doi: 10.1007/s10551-014-2416-0, Journal of Business Ethics, 1-13 (2014)

Rest, J., Moral Development: Advances in Research Theory, Praeger, Nueva York, USA (1986)

Rodríguez, M.P., Formación gerencial en valores. Unibiblos, Bogotá, Colombia (2008)

Rodríguez, M.P., Enfoque ético para la formación gerencial en uso de poder, en Formación gerencial en uso de poder: un enfoque ético, por M.P. Rodríguez, F. Cantor, M.A. Pantoja, J. Toro y A.F. Vásquez, pp 109145, Editorial UN, Bogotá, Colombia (2014)

Rodríguez, M.P., M.A. Pantoja y V. Salazar, Educación ética en ingeniería: una propuesta desde el currículo oculto, http://www.educacioneningenieria.org/index.php/edi/article/view/2/1, ISSN 1900-8260, Revista Educación en Ingeniería, 9, 104-116 (2010)

Rodríguez, M.P., F. Cantor, M.A. Pantoja, J. Toro y A.F. Vásquez, Formación gerencial en uso de poder: un enfoque ético, Editorial UN, Bogotá, Colombia (2014)

Sánchez, A., Ética, Random House Mondadori, México D.F., México (2005)

Schein, E.H., Organizational culture and leadership, Jossey-Bass, San Francisco, USA (2004)

Schmidt, J.A., Changing the paradigm for engineering ethics, doi: 10.1007/s11948-013-9491-y, Science and Engineering Ethics, 20, 985-1010 (2014)

Tashman, P. y J. Raelin, Who and What Really Matters to the Firm: Moving Stakeholder Salience Beyond Managerial Perceptions, Business Ethics Quarterly, 23(4), 591-616 (2013)

Tello, C. y M.P. Rodríguez, Categorías conceptuales para el estudio de la responsabilidad social organizacional, Hallazgos, 11(22), 119-135 (2014)

Treviño, L.K., Ethical decision making in organisations: A person-situation interactionist model. Academy of Management Review, 11(3), 601-607 (1986)

Tugendhat, E., Lecciones de ética, Gedisa, Barcelona, España (1997)

Vásquez, A.F., Actividades para la formación gerencial en uso de poder, en Formación gerencial en uso de poder: un enfoque ético, por M.P. Rodríguez, F. Cantor, M.A. Pantoja, J. Toro y A.F. Vásquez, pp 147-195, Editorial UN, Bogotá, Colombia (2014)

Warren, D.E., J.P. Gaspar y W.S. Laufer, Is Formal Ethics Training Merely Cosmetic? A Study on Ethics Training and Ethical Organizational Culture, Business Ethics Quarterly, 24(1), 85-117 (2014)

Weber, M., Economía y Sociedad. Tomos I y II, Fondo de Cultura Económica, México D.F., México (1977) 